\title{
EL MODELO CASOS Y CONTROLES PARA UNA EPIDEMIA LLAMADA MATEMÁTICAS BÁSICAS
}

\author{
Luz María Rojas Duque, José Gerardo Cardona Toro \\ Fundación Universitaria del Área Andina, Colombia \\ Universidad Tecnológica de Pereira, Colombia \\ lmrojas@,funandi.edu.co
}

El fracaso de los estudiantes que llegan al primer semestre de las carreras universitarias en Colombia se ha vuelto una epidemia, pues estos jóvenes presentan problemas conceptuales muy grandes en aritmética y álgebra, ocasionando con ello una enorme deserción, El presente trabajo muestra mediante un modelo epidemiológico de casos y controles la gravedad de este problema. Se tomaron para ello datos reales de las universidades de la región cafetera y se proponen al final estrategias que han demostrado reducir esta epidemia.

The failure of students arriving to the first semester of university courses in Colombia has become an epidemic, as these young people have very large conceptual problems in arithmetic and algebra, thereby causing a huge desertion this paper shows by an epidemiological model the seriousness of this problem. It takes real data from universities in the coffee region and intend to end strategies that have been shown to reduce this epidemic.

\section{INTRODUCCIÓN}

En el año 2012 motivados por la gran deserción que vienen presentando las universidades de la región y sobre todo las de nuestra localidad Pereira, Risaralda Colombia, nos interesamos a estudiar los motivos por los cuales, tantos jóvenes perdían matemáticas I y matemáticas básicas, estos datos los llevamos a un modelo epidemiológico y luego mediante una anamnesis, fuimos conociendo las diferentes causas, que llevan a estos jóvenes a fracasar en lo que ellos quieren estudiar como ingeniería, administración, economía e incluso derecho. El enfoque epidemiológico utilizado fue de riesgo relativo en casos y controles, el riesgo se define como la probabilidad de ocurrencia en una enfermedad (para nuestro caso es la mala preparación en aritmética y algebra), Existen diferentes riesgos: riesgo para ocupación, riesgo en función de exposición a factores de riesgo y riesgo competitivo. Con este modelo logramos obtener cuales eran los factores de riesgo más frecuentes en los jóvenes y sus cusas; entre ellos la mala enseñanza en las escuelas secundarias, pues desde que en Colombia se dejo de evaluar a los estudiantes bajo la escala numérica y adoptando un modelo de notas cualitativa y dando miles de opciones y bajo los estándares requeridos el nivel de evaluar termina siendo poco exigente hace que al final aprueben cursos con conocimientos netamente básicos y sin mayores exigencias. Los estudiantes se han relajado y como saben que ganan de cualquier manera, le prestan poca atención a los cursos y sobre todo a los de matemáticas, por ello se han adoptado en las universidades en los primeros semestre diferentes opciones y estrategias buscando que los estudiantes se queden y puedan sobresalir, pero no se había realizado un caso control donde se enfocara en su vida familiar, los conceptos básicos de la aritmética y el álgebra, Estableciendo para ello un control de vigilancia a cada estudiante donde se involucre su acudiente más cercano. Es importante decir que si no hay una buena conceptualización matemática no habrá buena interpretación de resultados estadísticos futuros, pues muchos de estos estudiantes en un 90\% deberán cursar asignaturas como: estadística descriptiva, inferencial y muestreo, por ello la importancia de dar al estudiante una seguridad en sus conceptos matemáticos y fortaleza al momento de tomar decisiones estadísticas.

\section{Marco teórico}

Los trabajos realizados por (Posso, 2008) encontraron diferentes causas sobre los problemas de la deserción por matemáticas y el fracaso del programa GEMA el cual busco mediante la conformación de seminarios y tutorías la corrección de las falencias encontradas, pero desafortunadamente el modelo pedagógico no funcionó y los estudiante decidieron desertar y no asistir más a las reuniones académicas planteadas, los demás estudios realizados se encontraron en

In: M.A. Sorto (Ed.), Advances in statistics education: developments, experiences and assessments. Proceedings of the Satellite conference of the International Association for Statistical Education (IASE), July 2015, Rio de Janeiro, Brazil. 
artículos sobre cómo mejorar las competencias matemáticas en estudiantes de ingeniería (Mora y Bueno, 2007)de grado de pregrado en la licenciatura de matemáticas y física, pero que solo pasó de un simple trabajo de datos estadístico, sin atacar de raíz el problema. Esto nos motivó a que se formara una investigación que encontrara las razones y se modelara, se controlará y además se propusieran soluciones. Las matemáticas son un elemento fundamental para entender los conceptos estadísticos y las salidas de los resultados que arrojan los programas estadísticos, la teoría de la probabilidad tiene como base fundamental el manejo matemático riguroso en conceptos de estimación y en general de la inferencia estadística, por ello decidimos atacar la base del problema la matemática básica. Hubo una serie de hallazgos que permitieron conocer como los estudiantes no manejan operadores como $\sum_{i=1}^{n} f(i)$ y $\prod_{i=1}^{n} f(i)$, al momento de despejar cualquier incógnita para calcular un valor de la mediana de datos agrupados o los cuartiles, deciles y percentiles, observamos la gran dificultad en el tema aritmético y algebraico.

\section{Metodología}

Para el desarrollo de la investigación se pidió la colaboración tanto de estudiantes como de docentes, solicitando a los segundos que aplicaran una anamnesis y un test de conocimientos del cual se extrajo la información para así construir el modelo de riesgo y luego se buscará una solución al problema. La población objetivo de estudio fueron las universidades de Pereira, estudiantes del primer semestre, 2000 estudiantes, la muestra se obtuvo utilizando muestreo aleatorio de tipo proporcional obteniéndose una muestra de aproximadamente 500 estudiantes, durante 2 años. Se pidió ayuda a docentes que facilitaban informes del rendimiento académico en matemáticas de los estudiantes. Para analizar la información se utilizó una base de datos de Excel, se realizaron encuestas y anamnesis y se utilizó la fotografía para detectar errores los cuales se les mostraron a los estudiantes y se analizaron con ellos para luego mostrar sus correcciones, de estos errores se hizo un banco de ellos.

\section{Análisis de datos}

En general, hay cierto indicio de que el factor de riesgo sea una causa cuanto mayor sea la razón del riesgo del grupo a perder matemáticas I o Básicas con respecto al grupo no expuesto a riesgo, por tener menos conocimientos y una muy débil preparación en sus bases aritméticas y algebraicas. Al tomar una muestra representativa en el estudio de una población en un área dada, con un tamaño adecuado a la frecuencia del evento que se estudia, la distribución de dicho evento en esta muestra es similar a la de la población que toma como referencia. Se utilizó una tabla de doble entrada para obtener información de asociación entre dos eventos, con ello se pretende buscar cuantos individuos tienen una falla $\mathrm{E}+\mathrm{y}$ cuantos tienen una frecuencia $\mathrm{Fr}+$. Esta asociación por si, no indica causalidad, pero da bases a posteriores estudios para averiguar si $\mathrm{Fr}+$ es un factor de riesgo para E+ y si hay suficiente fuerza para pensar en causalidad, después de controlar otros factores asociados y hacer inferencia.

Porcentaje del riesgo atribuible al factor de riesgo (RA\%), está dado por:

$$
R A \%=\frac{\left(\frac{60}{1000}\right)-\left(\frac{3}{1000}\right)}{\frac{60}{1000}} \times \frac{100}{100}=0,95
$$

Esto nos indica que el 95\% de los estudiantes está expuesto al factor de riesgo es decir a perder la asignatura de matemáticas básicas o matemáticas I, por los factores de riesgo expuestos, es decir el poco conocimiento de aritmética y algebra, el 5\% restante se debe a factores como escaso trabajo extraclase o problemas de otra índole, bajo interés por la carrera que están estudiando o por que no encontraron dinero para estudiar en el extranjero. (Calero, 1989) 


\section{ESTIMACIÓN DE PUNTO DEL RIESGO RELATIVO}

En el estudio realizado para "casos y controles" se toman dos grupos, uno en el cual se somete a continuas tutorías, ayudas como el cuaderno de taller, videos y acompañamiento, el otro solo se deja en libertad al estudiante que se muestra un poco reacio o el supone que tiene muy buen conocimiento, para lograr el estudio de estos casos se realizó una razón de disparidades u odds ratio.

Se llamó a los estudiantes con muy poca asistencia a las tutorías se tomaron 200 estudiantes tanto para el grupo A como para el grupo B.

En un riesgo alto como el A encontramos un 10 por 1000 y cuya distribución de factor de riesgo es del $75 \%$. Con el supuesto de la población ayudada es decir la B el factor de riesgo positivo es de $25 \%$, para una población de 2000 estudiantes.

En la tabla 1 se muestra la estimación de riesgo relativo.

Tabla 1 Análisis para la estimación del riesgo relativo.

\begin{tabular}{|c|c|c|c|}
\hline 1 & 2 & 3 & 4 \\
\hline & E+ & E- & Total \\
\hline Fr+ & 16 & 484 & 500 \\
\hline Fr- & 4 & 1496 & 1500 \\
\hline Total & 200 & 1980 & 2000 \\
\hline
\end{tabular}

Realizando los cálculos correspondientes, se encontró:

Tabla 2 Análisis con base en Probabilidades

\begin{tabular}{|c|c|c|c|}
\hline 1 & 2 & 3 & 4 \\
\hline & E+ & E- & Total \\
\hline Fr+ & 0,008 & 0,242 & 0,25 \\
\hline Fr- & 0,002 & 0,748 & 0,75 \\
\hline Total & 0,01 & 0,99 & 1 \\
\hline
\end{tabular}

Por lo tanto el riesgo relativo está dado por:

$$
R R=\frac{\frac{0,008}{0,25}}{\frac{0,002}{0,75}}=12
$$

Este resultado nos muestra que hay un gran riego relativo el cual debe ser controlado con tutorías, acompañamientos, cuaderno de talleres y test de conocimientos para así ir bajando la deserción. Utilizando términos de probabilidad, la razón de disparidades o estimación del riesgo relativo, odds Ratio, será:

$O R=\frac{0,008 \times 0,748}{0,242 \times 0,002}=12,36$ es un valor que muestra que existe un riesgo latente de deserción si no se realiza un acompañamiento con vigilancia a cada caso control de los estudiantes involucrados en los planes de trabajo; es decir no basta con prestar las tutorías y acompañamientos si no se está revisando continuamente el avance en conceptos de los estudiantes y esto se logra con la continua evaluación mediante exámenes de desempeño. 
E1 IC del 95\% encontrado fue: $(5,21,29,35)$ que fue muy significativo ya que no contiene el valor de 1,0; quiere decir que existe un valor muy alto de riesgo si no se hace una vigilancia completa a cada estudiante de sus errores en los exámenes individuales, programados.

\section{ESTRATEGIAS}

Para poder aplicar en contexto lo analizado es muy importante seguir las siguientes recomendaciones:

1. Antes de iniciar el curso de matemáticas básicas se hizo necesario realizar una anamnesis, con la cual se logra descubrir de donde viene el estudiante, su conformación hogareña, su época infantil, juvenil y sus etapas escolares antes de llegar a la universidad, el colegio donde egresó.

2. Hacer una prueba diagnóstica de conocimientos, en la cual el estudiante muestre su nivel de conocimientos en aritmética y algebra, con ella sabremos si está en condiciones de asumir el curso de matemáticas básicas o si se le debe nivelar, utilizando para ello un docente, un monitor y la ayuda de sus padres.

3. Realizar una muy buena selección de ejercicios para tener un banco que luego pueda ser abordado por el estudiante como medio para que él pueda entrenarse para futuras pruebas, además, con estrategias pedagógicas en las cuales haya constantemente recuerdos de los conceptos y problemas de entrada, intermedio y superior, para con ello ver el autoaprendizaje, la superación en conceptos y aplicaciones de los mismos, autoevaluación y evaluación y sobre todo lo más importante responsabilidad personal.

4. Se deben formar grupos de trabajo en los cuales haya discusiones y que los estudiantes de avanzada refuercen a sus compañeros.

5. A los estudiantes destacados se les sugiere a los docentes se les tenga en cuenta bonificaciones y otras compensaciones, para que exista motivación y el estudiante sienta que su trabajo, aprendizaje y apoyo a sus compañeros sea un aliciente como persona.

6. Se deben mostrar las aplicaciones de la matemática en la vida cotidiana y las diferentes ciencias, para fomentar más aprecio por ellas.

La prueba diagnóstica se basó en conceptos de simplificación de fracciones aritméticas, factorización de expresiones algebraicas, simplificación de fracciones algebraicas, simplificación de expresiones radicales donde implicara la racionalización y conceptos fundamentales de funciones, como identificación de funciones, dominios y aplicaciones sencillas en diferentes áreas de la vida cotidiana.

\section{CONCLUSIONES}

En el trabajo se utilizaron métodos para analizar datos desde una perspectiva de la epidemiología y más exactamente el riesgo relativo y casos y controles, tablas 1 y 2 . Es muy importante para lograr una disminución de la deserción de los estudiantes de primero y segundo semestre, tomar medidas como implementar talleres, tutorías, acompañamientos por parte del docente, el cuaderno de talleres, las estrategias utilizadas y algo muy importante la anamnesis, $\mathrm{n}$ esta se debe preguntar nombres y apellidos, edad, colegio donde estudió, composición de su familia, horas dedicadas a estudiar matemáticas, trato con sus padres, acompañamiento familiar entre otras, que da un informe muy interesante sobre la vida familiar y académica del estudiante.

Con este estudio desarrollado, se pudo presentar un informe a las diferentes instituciones, el modelo de riesgo fue adoptado y viene aplicándose con el fin de ir estudiando la deserción. En algunas instituciones se crearon grupos llamados antidesercción, ya que la deserción llegó incluso a valores del $75 \%$.

Con las estrategias planteadas los cursos han crecido y su deserción es menor, además hay más compañerismo, respeto y amor por las matemáticas.

\section{REFERENCIAS}

Calero, J. D. (1989). Método Epidemiológico. Madrid: McGraw Hill.

Enrique, P. A. (2008). Si son operaciones básicas, ¿por qué es tan difícil? Entre Ciencia e

Ingeniería, 138 a 156. 
Mora, D. M., y Bueno, r. B. (2007). Sistema pedagógico para el aprendizaje exitoso de las matemáticas. Bogotá: Universidad Central; Colombia. 